\title{
Research on Computer Algorithm and Display Method of Network Diagram
}

\author{
Wangming Yang \\ College of Computer and Information, China Three Gorges University, Yichang, 443002, China
}

Keywords: Network diagram, Computer algorithm, Display method, Research.

\begin{abstract}
Information technology has a very important role in the development of human society. Human development plays an irreplaceable role in in the process of social production practice. Through various mathematical models built by the computer, solve a variety of problems appears. In order to solve some discrete problems, relying on computer numerical calculation will play an irreplaceable role. In this paper, we mainly discuss the computer algorithm and display method of network diagram, and make some brief descriptions on some basic concepts of network diagram. The development process of computer algorithm and display method of network diagram is briefly introduced and the future is fully prospected, its purpose is to make science and technology have better development.
\end{abstract}

\section{Introduction}

The network diagram of general sense, which means a model with a diagram. The general network diagram consists of operation, events and routes. With the development of the times, human society has entered the information society, the application of network diagram has become very wide, and it is frequently applied in the communications network, process flow, circuit networks, and other fields. The extent to which the network graphs depend on the different supply and demand in the project can have a good display and can make it a whole. In general, the network map can be a good show of the entire work plan, and after programming, the key part of the whole work is a good embodiment, which can reduce the construction of repeatability.

Science and technology professionals will firstly conduct qualitative analysis on dispersion degree of some original data in the network diagram, and then conduct quantitative analysis, so that we can get further optimization program. In addition, the relationship between the edge and the vertex of the edge of the relationship between the inferred, so as to determine the connectivity between the graph and the shortest path.

In the actual application of the network diagram, for different computer algorithms, there are different service objects, which requires us to strengthen the research work on the algorithm, making the technology, computer theory and network diagram of the control algorithm for effective combination of services other areas, to make greater and better contribution for the development of human society.

\section{Control Algorithm of Network Diagram}

The application of network diagram in human life is very extensive. Through the research and application of the full control algorithm of the dot symbol and the control algorithm of the edge symbol, technical persons can get the new network diagram network.

\section{Development process of point symbol full control algorithm}

The theoretical basis of point symbol and the full control algorithm are commonly developed. The point symbol full control algorithm is combined with symbol control algorithm, adding a certain limit, and the maximum and minimum values are specified. For this algorithm, in the lower limit, you can achieve the lower bound between each other to update this purpose. In the process of the image, the lower boundary to update the problem, on the consideration, you can get the conditions of the 
network map for the network map full control algorithm, must meet the requirement that the symbol must be comprehensive and complete. After entering the twenty-first century, Zelinka first proposed the concept of symbol control algorithm, from that time on, symbol control algorithm began to be studied. In 2003, Xing Huaming and his team gave a definition for the lower bound of the full sign algorithm. In 2004, Michael A Henning found a more specific network map to meet these lower bounds. In 2006, Wang Junxiu for the actual situation, the same point on the symbol control algorithm for a certain amendment, which gives a lower than the original lower than the new lower bound. In 2009, Lv Xinzhong and his team complement the full control algorithm of the network graph to make the content more complete. After the improvement of the network map is more and more scholars of concern, which also contributed to the development of network plans better and faster.

\section{Development process of edge full control algorithm}

As early as 2001, Xu Baogen proposed the basic concept of edge symbol control algorithm, which makes the network diagram control algorithm has been greatly improved. In 2002, Bohdan Zelinka Liberec gave the problem of the bounds, and then $\mathrm{Xu}$ Baogen also gave the upper bound and the lower bound of the edge symbol control algorithm, and gave a more accurate value for the partial number of the edge symbol control algorithm. For the reduced control algorithm, it is another form of edge symbol control algorithm, but for the reduction control algorithm, the difficulty of research is very large, and the research time is relatively short, so the relevant In addition, there is no research results, which requires the relevant technical staff to strengthen the study of the reduction control algorithm.

\section{Impact of point symbol, edge symbol control algorithm development on science and technology development}

For point symbol and edge symbol, the development of its control algorithm for the network diagram of the display system and the query system, there are many benefits. It should be noted, however, that this kind of inquiry system is very easy to change during the course of work, especially when the system has been changed many times, if you want to know the operation record, it becomes very difficult, the image of the display will be very unclear. Therefore, when you have to point symbol control algorithm and edge symbol control algorithm to modify the time, should be fully with the role of the database, in order to make up for the point symbol and edge symbol control algorithm deficiencies, and thus can make the problem of data query Effective solution.

\section{On the improvement of computer algorithm of network diagram}

On the issue of computer algorithms, since entering the twenty-first century, science and technology workers have put forward new demands. As early as 2009, Lv Xinzhong and Yi Mingyuan had found a control algorithm of the symbols that could get the special network graph of the centralized type, which further enhanced the relevant content of the symbol control algorithm. In fact, before this, people have begun to use anti-sign algorithm, and in these years have made anti-symbolic algorithm into anti-symbol full control algorithm of a trend, which has become the direction of science and technology workers in recent year's efforts. In addition, the world symbolic algorithm is further improved at the same time, many scientific and technological workers through practical research, found a special network symbol edge control algorithm to determine the specific value, so as to determine the general network map boundary. According to the existing theoretical results and practical conditions, the reduction control algorithm still has some difficulty. After the full research, the research direction of the next scientific and technological workers should be in the K-side control algorithm, and some specific requirements Is to let the edge reduction control algorithm required to meet the "all sides" of the conditions into "at least K side." However, we need to note that this study also has a certain degree of difficulty, for now, this project is still in the initial research stage. 
In addition, the reason why people want to study the shortest path, the purpose is not only to make the accuracy of the algorithm is guaranteed, but also to achieve the algorithm to make the search space to narrow the purpose, which can make the computer load capacity is improved, And ultimately achieve the purpose of improving the efficiency of computer operation. When the computer's operating efficiency is greatly improved, the benefits of the calculation of the network map can be very simple, the shortest path algorithm related to the complexity of the calculation will change with the network size will be the corresponding changes. In the general network, the number of nodes $n$ and the number of even the edge of $m$ there is a great relationship between, so people in the actual application of the network process, through the way to reduce the order to build the network Of the hierarchical model, making the network topology is simplified, and thus reduce the complexity of the algorithm to achieve the purpose. In the network, the number of nodes and the number of edges will be reduced due to the increase in the hierarchical level of the network. Therefore, by switching to high-level topological wear, the number of nodes that exist in the calculation process Get effective to reduce this, to improve the efficiency of the computer algorithm, there is a very big effect. In the shortest path of the algorithm process, if the search process without any purpose, it will make the search there is a very large waste of resources. If the application of the network, the use of accelerated guidance technology, making the number of nodes in the calculation process is reduced, making the complexity of the algorithm is reduced. In order to improve the computational efficiency and accuracy of the shortest path algorithm, it is also possible to work on the search side. In the actual application process, the optimization of the search strategy can not only get a faster path response speed, so that the calculation accuracy of the path is improved, and the consumption of the storage space can be reduced, and the versatility of the algorithm can be improved.

\section{Display methods of network diagram and main algorithm}

\section{Theoretical basis of computer display of network diagram}

For the computer display of network diagram theory, mainly use $\mathrm{C}$ language to achieve the drawing of the network diagram, which contains two reasons: First, the $C$ language is very simple and very powerful, which makes it very easy to draw on the big screen; Second, use C language for programming, it uses very small memory and it has a very strong implementation efficiency. The relationship between the vertex and the edge is very complicated in the process of analyzing the connection of the point of the network graph. For science and technology workers, in the process of drawing the network map, they must first draw each vertex, followed by each vertex to use the line to connect. Staff in the use of $\mathrm{C}$ language for drawing time, the general is often used in physical coordinates, for the horizontal direction, the provisions of the $\mathrm{X}$-axis, and the right to the positive direction, left to the negative direction. For the vertical direction, it is specified as the Y-axis, and it is the positive direction, and the downward direction is the negative direction. The staff in the use of cheating $(\mathrm{X}, \mathrm{Y})$ that each point, the requirements of $\mathrm{X}, \mathrm{Y}$ must be as an integer to appear, and also need to have a certain range of values.

Once the diagram is drawn, it is necessary to make the size and position of the graphic appropriate, and to zoom, pan and rotate the image as necessary. After the process of displaying the algorithm of the graph is completed, the network diagram is controlled, added or reduced, and the time is recorded in the course of historical inquiry.

\section{Steps required of algorithm to finish diagram}

(1) The technician draws the complete graphic on the screen through the information given by the user.

(2) When the user's information is entered, the corresponding image is obtained according to the necessary information. In particular, it is necessary to use other colors to describe the re-added edge. 
(3) Through the user to add a new point to form a map, the information needs to include a variety of information on the edge, and finally draw the image, the use of other colors to achieve the re-add points and edges to describe.

\section{Notices in the network diagram display images}

When the staff in the process of operating the network map, the input data and add points and other details of the problem to pay special attention. When inputting the data, the input should be done first, followed by other information. In other information, there are generally the number of vertex and its number, the number of edges and the number. When the edge and the point of the input, the first input to the command, then the specific number of input. In general, the relevant bibliography should include the starting position of the edge and the number of end points, and also numbered, the adjacent multi-table changes, so as to complete the drawing of the network map. For the network diagram in the computer algorithm and the actual display process, its basis should be the point symbol control algorithm and edge symbol control algorithm. When the application of the actual control algorithm for the network diagram, we must constantly improve the design of the network diagram, so that the work efficiency of the computer continues to improve.

\section{Conclusions}

Into the twenty-first century, the development of science and technology is getting faster and faster, the development of the theory of network diagram is getting faster and faster. The research of network graph theory has many problems in the structure and relationship between discrete things Benefits, can make qualitative or quantitative aspects of the problem is greatly optimized, so that the problem of solving the process becomes more rigorous, digital. Using points and lines to solve the problem, you can solve many problems by relying on the establishment of mathematical models, so that the problem is solved more simple. However, we need to note that the current industrial control in China is moving towards the direction of intelligent and open, on the one hand, to the user to bring a very large convenience colleagues, on the other hand, there is still With a certain security risks. For example, when there is foreign data into the time, PLC does not have the necessary verification of foreign data, which makes the industrial Ethernet there is a very large security vulnerabilities. From this level, how to make the system more perfect function, how to become more accurate computer algorithms, making the industrial control system more secure, we need to continue to study and explore. The rapid development of computer technology has promoted the rapid development of algorithm theory. In the next time, we should be toward the computer network, topology and coding theory in this direction, so that the network map can be better for the development of human society to contribute.

\section{References}

[1] Gao Fu. Computer Analysis of Computer Algorithms Based on Network Diagram, Electronic Technology and Software Engineering, 2014, 54(21), 20.

[2] Xu Jin. Research on Computer Algorithm and Display Method of Network Diagram, WENLIDAOHANG, 2015, 45(5), (3rd issue), 6.

[3] Wang Lingyan. Research on Computer Algorithm and Display Method of Network Diagram, Electronic Technology and Software Engineering, 2015,89(1), 189-190.

[4] Wang Li. Research on Computer Algorithm and Display Method of Network Diagram, Journal of Shandong Agricultural Engineering Institute, 2015, 56(3), 42-43,46.

[5] Wu Kai, Li Peng, He Yongqiang. Research on Control Algorithm Theory and Network Diagram Computer Algorithm Display, Modern electronic technology, 2015, 17, 151-152, 157. 